\title{
Direct Detection Electron Energy-loss Spectroscopy: Applications in Low-dose Chemical Mapping and In Situ Heating+biasing
}

\author{
Mitra L. Taheri ${ }^{1}$, James L. Hart ${ }^{1}$, Andrew C. $\operatorname{Lang}^{1}$
}

1. Department of Materials Science \& Engineering, Drexel University, Philadelphia, PA 19104, USA

In situ transmission electron microscopy (TEM) and electron energy-loss spectroscopy (EELS) are powerful tools for the observation of real-time materials processes. The development of radiation hard direct detection (DD) electron sensors has enabled improvements in the quality of in situ data for TEM imaging, and recently we demonstrated that DD provides similar benefits for EELS [1]. Specifically, the sharper point spread function and reduced pixel size of DD provides a significant improvement in combined energy resolution and field of view (FOV) (Fig. 1a). This combined resolution/FOV facilitates simultaneous elemental mapping (large FOV) and oxidation state analysis (high resolution). Additionally, the improved detective quantum efficiency (DQE) offered by electron counting greatly reduces spectrum noise, which has benefits for low-dose applications and time-resolved in situ studies (Fig. 1b).

Here, we review some of our group's recent work with DD EELS: in situ investigation of MXenes and low-dose chemical mapping of mouse cervical tissue. MXenes are a growing material family with the chemical formula $\mathrm{M}_{n+1} \mathrm{X}_{n} \mathrm{~T}_{\mathrm{x}}(\mathrm{M}=$ transition metal, $\mathrm{X}=\mathrm{C}$ and/or $\mathrm{N}, \mathrm{T}=$ surface termination, e.g. $-\mathrm{O},-\mathrm{F}$, $-\mathrm{OH})$. These materials show exceptional performance in areas such as energy storage and electromagnetic interference shielding [2]. Density functional theory predicts a strong influence of terminations on electronic properties, which could allow surface chemistry engineering for improved material performance. However, experimental confirmation of these predicted chemistry-property relations is lacking. Through in situ DD EELS, we provided the first direct correlation of MXene terminations and conductivity. Figure 2 shows that a decrease in $-\mathrm{F}$ termination of $\mathrm{Ti}_{3} \mathrm{C}_{2} \mathrm{~T}_{\mathrm{x}}$ produces a sizable increase in conductivity. We have additionally studied several other MXenes, the role of different terminations, and intercalation. These results both advance our basic understanding of MXene properties and provide guidelines for the optimization of MXene devices. For these experiments, the improved energy resolution/FOV of DD EELS was necessary to observe all relevant elements (C through F) and maintain sufficient energy resolution.

Preterm birth is a major health issue which continues to contribute to acute and long-term neonatal morbidity. Our understanding of the underlying causes of preterm birth is still unclear, though the mechanical properties of the cervix are thought to play a role. To understand this problem, researchers can study the cervical tissue of mice [3]. While it is common to perform electron microscopy of such samples, electron energy-loss spectroscopy is challenging due to the high beam sensitivity of biological specimens. Here, we use DD EELS and DD EFTEM to study the chemical structure of non-pregnant and pregnant mice. The improved DQE of DD allows a greatly reduced electron dose which is critical for the study of these sensitive samples.

In summary, DD EELS is a relatively new technique which improves the combined energy resolution and FOV and increases spectrum SNR. We have utilized DD EELS to perform in situ measurements of MXene samples and low-dose chemical analysis of biological tissue. 
References:

[1] J.L. Hart et al., Sci. Rep. 7 (2017), p. 8243

[2] B. Anasori, M.R. Lukatskaya \& Y. Gogotsi, Nat. Rev. Mat. 2 (2017), p.16098

[3] M. Mahendroo, Reproduction 143 (2012), p. 429

[4] JLH, ACL, and MLT acknowledge funding from NSF MRI grant number 1429661.

a)

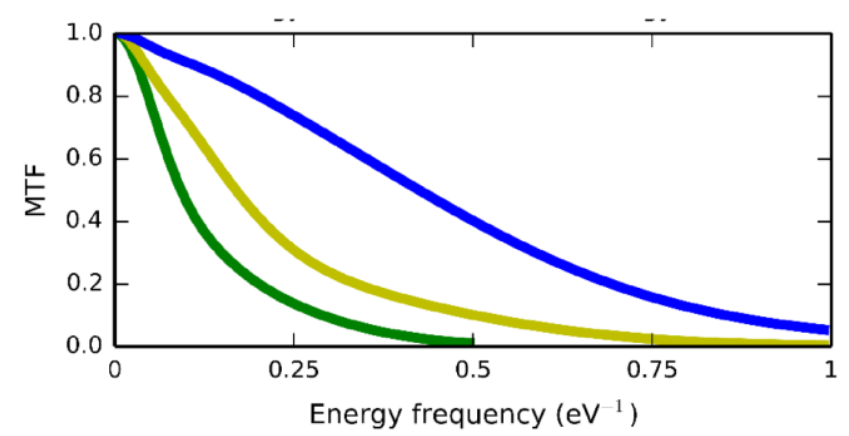

b)

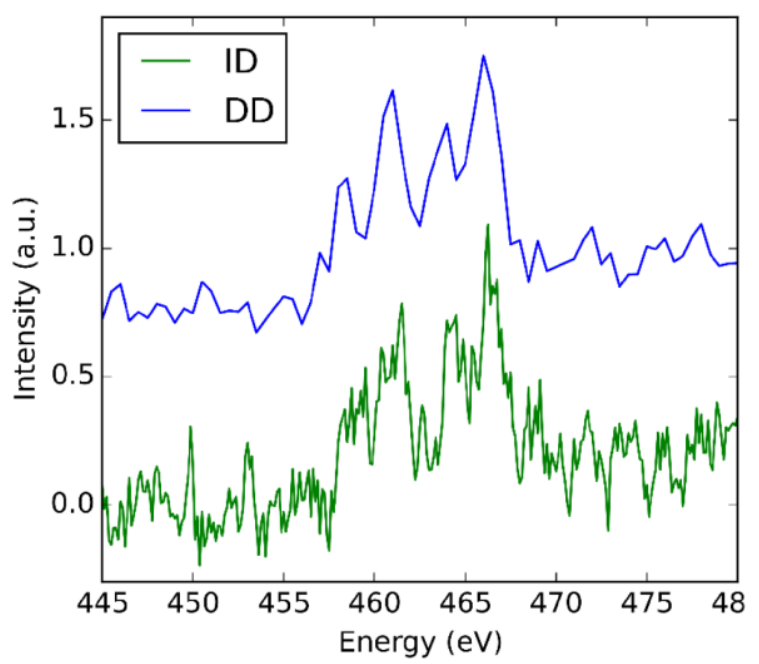

Figure 1. a) Modulation transfer function (MTF) of a DD EELS system at $0.5 \mathrm{eV} / \mathrm{channel}$ dispersion and $2 \mathrm{k}$ eV FOV plotted against a convention indirect detection (ID) EELS system. The MTF was obtained by taking the Fourier transform of the measured zero loss peak. b) Comparison of a Ti L edge from the $\mathrm{SrTiO}_{3}$ sample acquired with DD EELS and conventional EELS. The improved SNR of DD EELS is evident.
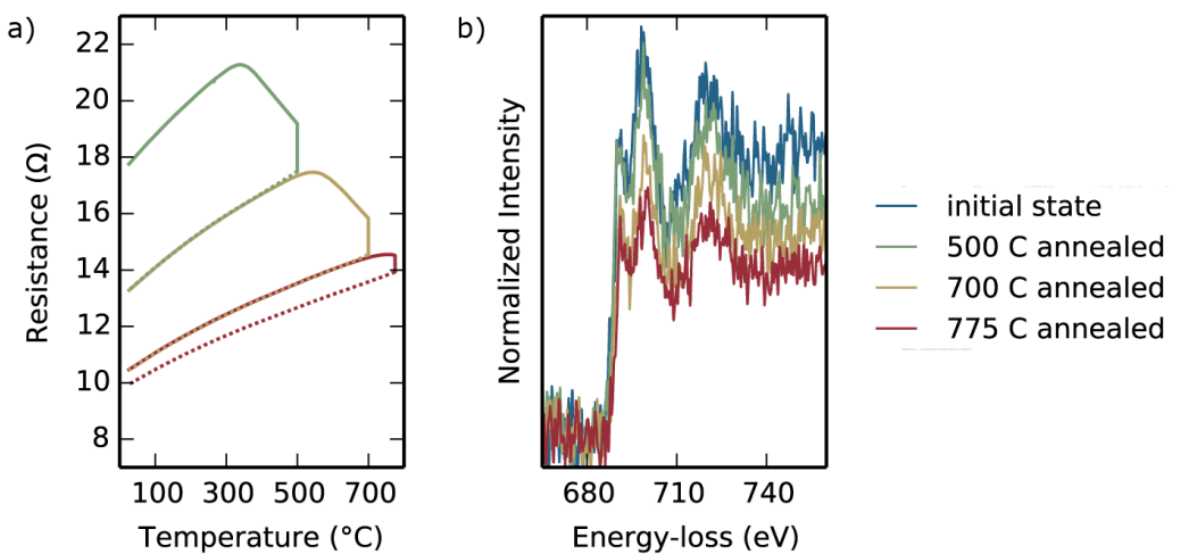

Figure 2. a) Resistance as a function of temperature for several annealing steps of a $\mathrm{Ti}_{3} \mathrm{C}_{2} \mathrm{~T}_{\mathrm{x}}$ sample. Each annealing step is shown with a different color. Solid line is heating, dotted line is cooling. b) EELS spectra taken before and after each annealing step shown in (a). Annealing lead to loss of $-F$ terminations and an increase in conductivity. 